\title{
Cáncer colorrectal de presentación atípica
}

\author{
Demian A Fullerton ${ }^{1}$, Francisco López $K^{1}$, \\ Rodolfo Avendaño $H^{1}$, Rodrigo Aparicio $R^{2}$, \\ Ignacio Wistuba $\mathbf{0}^{3}$.
}

\section{Atypical presentation of a colorectal carcinoma}

We report a 67 years old male with a history of pulmonary tuberculosis at the age of 15, that consults for malaise, weight loss and productive cough. Chest X ray examination showed a left pleural effusion. A pleural tap obtained a sterile exudate. A thorax CAT scan showed a proliferating mass in the splenic angle of the colon, with left lung and diaphragmatic invasion. Endoscopic biopsies confirmed the diagnosis of adenocarcinoma. The patient underwent a subtotal colectomy with partial excision of diaphragm and left lung. The pathological report showed a mucosecretory adenocarcinoma, infiltrating the pericolonic adipose tissue without adjacent organ infiltration and a chronic inflammatory process involving colonic serosa, diaphragm pleura, and lung. Adjuvant chemotherapy was indicated and the patient is asymptomatic and without evidences of tumor recurrence after a 24 months follow up (Rev Méd Chile 2004; 132: 985-88).

(Key Words: Adenocarcinoma, mucinous; Colonic neoplasms)

Recibido el 30 de enero, 2004. Aceptado el 22 de abril, 2004.

${ }^{1}$ Departamento de Cirugía Digestiva, ${ }^{2}$ Sección de Cirugía Torácica y ${ }^{3}$ Departamento de Anatomía Patológica, Pontificia Universidad Católica de Chile.

$\mathrm{P}$ aciente de sexo masculino de 68 años, quien refiere antecedentes de tuberculosis pulmonar diagnosticada y tratada a los 15 años y trastorno digestivo funcional desde hace 8 años. Consultó en Servicio de Urgencia por cuadro de 2 semanas de evolución de dolor tipo puntada de costado, acompañado de fatigabilidad, anorexia y baja de

Correspondencia a: Dr. Francisco López Köstner. Departamento de Cirugía Digestiva, Facultad de Medicina, Pontificia Universidad Católica de Chile. Marcoleta 367 Casilla 114-D, Santiago. Fono: 3546828-3543221. Fax: 6329620. E mail: flopez@med.puc.cl
$10 \mathrm{~kg}$ de peso en un mes. Al examen físico destacaba un paciente en buen estado general, afebril, con matidez y disminución del murmullo pulmonar en la base del hemitórax izquierdo. La radiografía de tórax confirmó el hallazgo de un derrame pleural. Se efectuó una pleurocentesis que concluyó un exudado inflamatorio, con adenosín deaminasa (ADA) normal $(14,1 \mathrm{U} / \mathrm{L})$, sin identificar gérmenes con la tinción de Gram ni células malignas en el estudio citológico. Se decidió continuar el estudio en forma ambulatoria, tomándose tres baciloscopias, que resultaron negativas. Por aparición de fiebre hasta $38,7^{\circ} \mathrm{C}$, se decidió su hospitalización para completar el estudio diagnóstico. 
Entre los exámenes de ingreso destacaron una anemia de 33,4\% de hematocrito, leucocitosis de 13.200, sin desviación a izquierda, VHS de $108 \mathrm{~mm} / \mathrm{h}$, proteína C reactiva de $18,4 \mathrm{mg} / \mathrm{dL}$ e hipoalbuminemia de 2,2 gr/dL Después del drenaje parcial del derrame con pleurocentesis, no se demostraron anomalías en la radiografía de tórax, sin embargo, el paciente persistió febril y reprodujo el derrame pleural. Se decidió iniciar terapia antibiótica empírica (con levofloxacino y cefotaximo) y apoyo con nutrición enteral. Se solicitó una tomografía axial computada (TAC) de tórax que evidenció una masa en el ángulo esplénico del colon que infiltraba, a través del diafragma, la base del pulmón izquierdo. Mediante colonoscopia se identificó una lesión proliferativa anular en el ángulo esplénico, que impidió el paso del colonoscopio a proximal. Se decidió completar la exploración del resto del intestino con una colonoscopia virtual, que no evidenció tumores sincrónicos. El estudio de biopsias endoscópicas demostró un adenocarcinoma de colon mucosecretor moderadamente diferenciado. En la TAC de abdomen y pelvis no se demostraron metástasis ganglionares ni a distancia. Se indicó la cinugía resectiva después de comprobar con ecocardiografía la ausencia de invasión al miocardio.

\section{Cirugía}

Se accedió al abdomen a través de una laparotomía media, en la exploración no había carcinomatosis peritoneal. Se observó una gran masa tumoral, que comprometía un área de aproximadamente $10 \mathrm{~cm}$ de diámetro en el diafragma, la que fue resecada en bloque con un segmento del lóbulo inferior del pulmón izquierdo (que se escindió disparando tres cargas de una corchetera lineal cortante) (Figura 1). Se instaló un tubo de pleurostomía y se cerró el defecto en el diafragma con puntos separados sin prótesis (Figuras 2 y 3 ). Se efectuó una colectomía subtotal habitual, con criterio oncológico, ligando las arterias cólicas y la mesentérica inferior en sus orígenes. Después de extraer la pieza operatoria, se efectuó una anastomosis íleo-sigmoidea manual. Se cerró la pared por planos y se maduró una ileostomía protectora en asa en la fosa ilíaca derecha. El procedimiento terminó sin incidentes en un tiempo operatorio de $240 \mathrm{~min}$, con una pérdida estimada de $600 \mathrm{cc}$ de sangre. La evolución postoperatoria fue satisfactoria, sin morbilidad quirúrgica, ni requerimiento de cuidados intermedios o intensivos, realimentándose con líquidos y retirándose el tubo pleural al

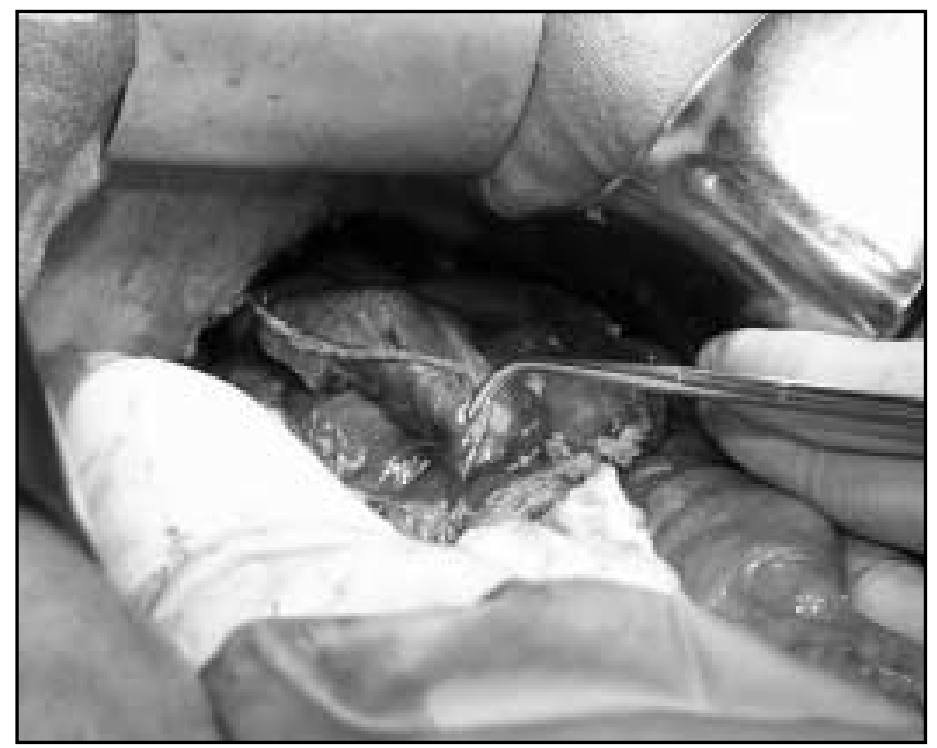

Figura 1. Disección del pulmón comprometido: Se observa el acceso al tórax desde el abdomen a través del diafragma. La pinza muestra el sitio de sección del pulmón con la corchetera automática cortante (stapler), el segmento pulmonar resecado se muestra adherido al trozo de diafragma que se resecó en bloc junto al colon. 

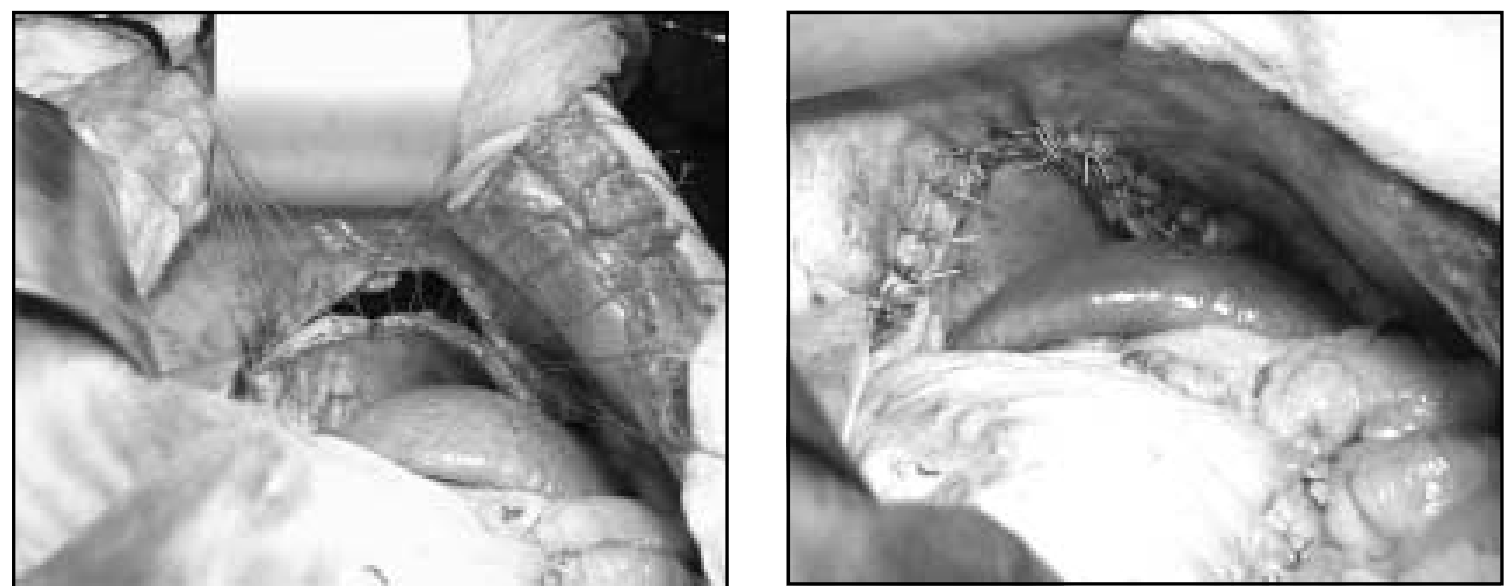

Figuras 2 y 3. Sutura del diafragma: Mediante puntos separados se suturó el defecto del diafragma después de la resección de un área de 9,5x7 cm que se encontraba adherida al colon. Se observan también el bazo y el estómago.

tercer día e indicándose el alta ocho días después de la cirugía. El cierre de la ileostomía se efectuó sin incidentes al mes y medio postoperatorio.

\section{Histopatología}

El estudio de anatomía patológica demostró un segmento de colon de $70 \mathrm{~cm}$, a cuya serosa estaba adherido un segmento de diafragma de 9,5 x 7 × 1 cm y un puño pulmonar de 9,5 x 4,5 x 2,7 cm (Figuras 4 y 5). La serosa del colon estaba hiperémica e indurada. La lesión tumoral era de aspecto nodular con zonas de aspecto mucinoso, con un tamaño de $16 \times 12 \times 3,5 \mathrm{~cm}$. El estudio microscópico demostró un adenocarcinoma de colon con $40 \%$ de componente mucosecretor, en el que el nivel máximo de infiltración llegaba al tejido adiposo pericolónico, a $3 \mathrm{~mm}$ del tejido muscular diafragmático (se examinaron múltiples cortes de la pieza incluyendo
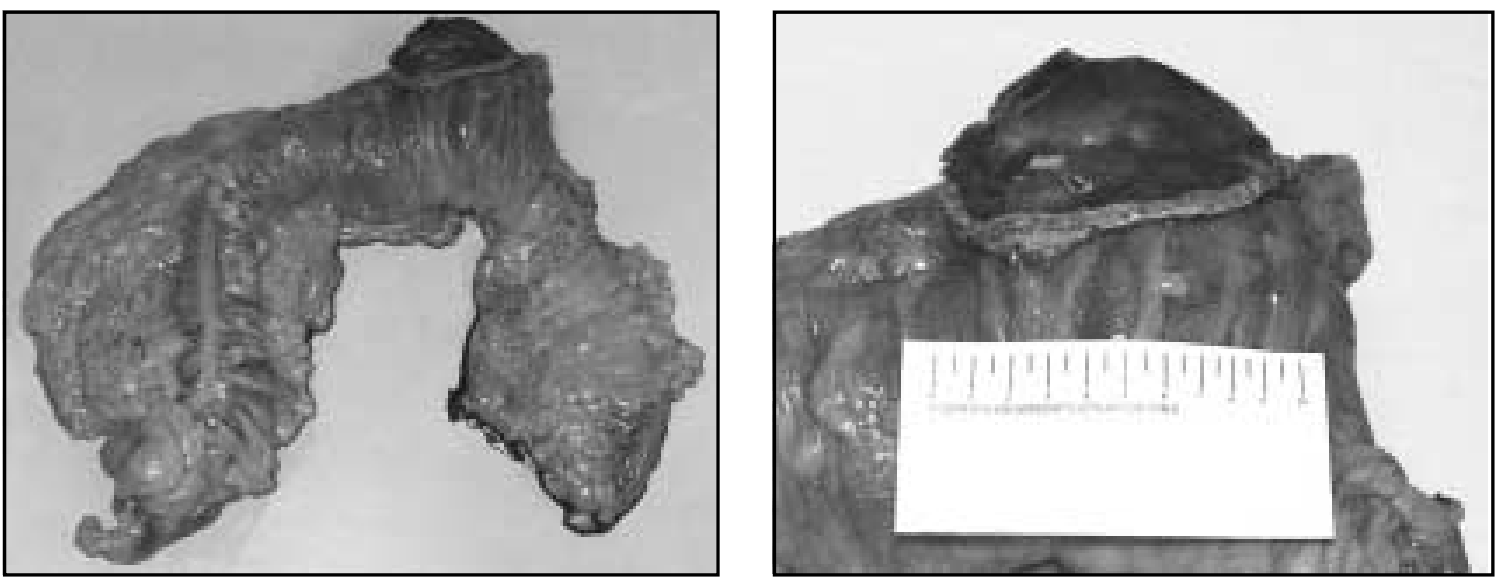

Figuras 4 y 5. Pieza operatoria: Se observa la pieza operatoria, por una colectomía total con resección de diafragma y neumonectomía parcial en bloc por adherencias de un cáncer colorrectal, cuyo estudio histológico demostró un proceso inflamatorio crónico sin infiltración carcinomatosa de los órganos resecados. 
diafragma, pericardio y pulmón, sin encontrarse infiltración). El compromiso de los órganos mencionados correspondía a un proceso inflamatorio crónico extenso con organización fibrosa y múltiples focos supurados que afectaban la serosa, el diafragma, las pleuras parietal y visceral y el pulmón. Se identificaron 102 ganglios linfáticos, cuyo examen histológico en ninguno demostró metástasis.

\section{EVOLUCIÓN Y SEGUIMIENTO}

De acuerdo al protocolo de tratamiento adyuvante en cáncer colorrectal de nuestro hospital, se indicó quimioterapia por tratarse de un cáncer de colon en etapa II con componente mucosecretor. El paciente ha sido controlado por 24 meses y no ha demostrado signos de recurrencia tumoral en el seguimiento que se ha efectuado con control clínico, medición en sangre del antígeno carcinoembrionario (CEA) y TAC de abdomen y pelvis anual.

\section{DisCUSIÓN}

Distintas series señalan que $10 \%$ de los casos de cáncer colorrectal ameritan resecciones multiviscerales por aparente infiltración. En estos casos, el porcentaje de pacientes que presentan efectivamente invasión tumoral de los órganos vecinos es $50 \% 1,2$. El resto corresponde a adherencias generalmente de tipo inflamatorio. El tratamiento de los tumores con adherencias a estructuras vecinas, requiere la resección en bloc de aquellos órganos aparentemente invadidos por tumor, sin fragmentar la zona de adherencia, pues se sabe que si se

\section{REFERENCIAS}

1. Lehner T, Methner M, PoLok A, Schaible A, Hinz U, HERFARTH C. Multivisceral resection for locally advanced primary colon and rectal cancer: an analysis of prognostic factors in 201 patients. Ann Surg 2003; 235: 217-25.

2. López M, Monafo W. Role of extended resection in the initial treatment of locally advanced colorectal carcinoma. Surgery 1993; 113: 365-72.

3. Hunter J, Ryan J, Schultz P. En bloc resection of separan los órganos adheridos del colon, o si existe incisión a través de la masa tumoral, existe una inaceptable tasa de recurrencia local y una pobre sobrevida ${ }^{2-4}$. Estudios demuestran que la sobrevida global, a cinco años, en pacientes sometidos a resecciones en bloc es comparable al de aquellos pacientes en que no se efectúan resecciones multiviscerales. Por otro lado, el pronóstico parece ser el mismo en los pacientes con estas cirugías, sin importar si la infiltración histológica de los órganos vecinos efectivamente ocu$\mathrm{rre}^{2,5,6}$.

En el Hospital Clínico de la Universidad Católica de Chile hemos reportado la experiencia en 30 pacientes. En la mitad de ellos existió infiltración de los órganos vecinos. Los tiempos operatorios fueron elevados (media: $4,5 \mathrm{~h}$ ) y obtuvimos una tasa de morbilidad de $30 \%$ y $3 \%$ de mortalidad, resultados que son comparables a series internacionales. Este estudio arrojó que $72 \%$ de los pacientes correspondían a etapa II, esto explica en parte la buena sobrevida que presentaron nuestros pacientes (una sobrevida actuarial específica por cáncer de $71 \%$ a 5 años). (Observaciones presentadas en el LXXVI Congreso Chileno de Cirugía).

Se sabe que en este grupo de pacientes, el pronóstico en sobrevida está determinado, antes que el compromiso por vecindad, por la presencia de metástasis linfáticas 5 . Es por esto que en el protocolo de terapia adyuvante de nuestro centro no se indica de regla quimioterapia en tumores localmente avanzados en etapa II, a menos que existan dudas en los márgenes quirúrgicos o bien por la presencia de otras caracteństicas de mal pronóstico, como son la presencia de componente mucosecretor que ocurrió en el caso que aquí presentamos.

colon cancer adherent to adjacent organs. Am J Surg 1987; 154: 67-71.

4. Gall F, Tonak J, Altendorf A. Multivisceral resections in colorectal cancer. Dis Colon Rectum 1987; 30: 337-41.

5. Poeze M, Houbiers J, Van De Veide C, Wobbes T, Von MEYeNFELDT M. Radical resection of locally advanced colorectal cancer. Br J Surg 1995; 82: 1386-90.

6. Gebhardt C MW, Ruckriegel S, Meier U. Multivisceral resection of advanced colorectal carcinoma. Langen becks Arch Surg 1999; 384: 194-9. 